\title{
Estratégia de Análise Quantitativa para Revisão de Pré-requisitos em uma Matriz Curricular do Curso de Bacharelado em Sistemas de Informação
}

\author{
Luciano Antonio Digiampietri ${ }^{1}$, Marcelo de Souza Lauretto ${ }^{1}$, Fábio Nakano ${ }^{1}$ \\ ${ }^{1}$ Escola de Artes, Ciências e Humanidades (EACH) - Universidade de São Paulo (USP) \\ São Paulo - SP - Brasil \\ \{digiampietri, marcelolauretto, fabionakano\}@usp.br
}

\begin{abstract}
The management of an undergraduate course includes tasks such as updating the curriculum and prerequisites, in order to provide a better professional background and to minimize the student dropout. This paper presents a strategy for quantitative analysis of association between disciplines, aiming to identify potential needs of new prerequisites in the course matrix. As the proposed approach is based exclusively on academic records, it is easily reproducible in any courses and universities, and has helped to modify some prerequisite in the Information Systems Undergraduate Course at USP.
\end{abstract}

Resumo. Dentre as estratégias de gerenciamento de um curso superior de qualidade, a atualização da estrutura curricular e dos pré-requisitos entre disciplinas podem contribuir para o melhor preparo do aluno para os desafios da vida profissional, bem como diminuir as taxas de reprovação e evasão. Este artigo apresenta uma estratégia de análise quantitativa de associação entre disciplinas, visando a identificar potenciais necessidades de revisões nos pré-requisitos adotados no Projeto Pedagógico do Curso. Por ser baseada exclusivamente nos históricos acadêmicos dos estudantes, a estratégia é facilmente replicável em quaisquer cursos e universidades, e tem auxiliado na alteração de alguns prérequisitos no curso de Bacharelado em Sistemas de Informação da USP.

\section{Introdução}

Um dos desafios permanentes no gerenciamento dos cursos de graduação é implementar e avaliar ações para aumentar qualidade e atratividade por meio da atualização da estrutura curricular, oferecimento de atividades extracurriculares, criação de programas de tutoria e apoio estudantil, entre outras.

O aperfeiçoamento da estrutura curricular é uma tarefa contínua que envolve a participação de coordenadores, professores e alunos. Diferentes estratégias vêm sendo relatadas ao longo dos anos para que esta atividade seja efetiva, desde a adaptação de currículos base internacionalmente aceitos [ACM and IEEE 2013], de forma a tornálos mais adequados a realidades locais ou para preencher lacunas existentes no mercado de trabalho [Outlay and Krishnan 2010]; a adaptação de ementas para torná-las mais atuais e/ou mais aplicadas de forma a serem mais atrativas aos alunos; e a estruturação de currículos mais flexíveis. 
Dentro do planejamento e da revisão da estrutura curricular, a adoção de prérequisitos entre disciplina desempenha um papel relevante, na medida em que busca garantir uma melhor base de conhecimentos ao aluno antes deste matricular-se em uma dada disciplina, permitindo assim um melhor aproveitamento e desempenho na mesma. Contudo, a introdução de pré-requisitos é usualmente feita exclusivamente com base nas análises de conteúdos entre disciplinas, sem o suporte de análises quantitativas de associação entre desempenhos em disciplinas.

A questão de pesquisa deste trabalho é o papel dos pré-requisitos como mecanismos para melhorar o aproveitamento e o desempenho acadêmico dos estudantes. Mais especificamente, a hipótese aqui estabelecida é de que o desempenho médio de um aluno em algumas disciplinas de níveis intermediários e avançados (oferecidas a partir do $2^{\circ}$ semestre) depende do desempenho desse aluno em disciplinas cursadas anteriormente. Nesses casos, pré-requisitos podem ser inseridos ou ajustados para explicitar ao aluno a necessidade da assimilação de ao menos parte do conteúdo da disciplina-requisito antes de matricular-se em uma dada disciplina, diminuindo assim suas chances de reprovação na mesma.

Com base na questão de pesquisa acima, o objetivo deste artigo é apresentar uma estratégia de análise quantitativa de desempenhos em disciplinas, visando a identificar potenciais relações de dependências entre disciplinas e, dessa forma, apoiar revisões nos pré-requisitos adotados no Projeto Pedagógico do Curso. Por ser baseada exclusivamente nos históricos acadêmicos dos estudantes e em estatísticas de fácil obtenção, a estratégia é facilmente replicável em quaisquer cursos e universidades.

Neste trabalho, apresentamos uma aplicação detalhada da estratégia proposta sobre os históricos acadêmicos dos alunos do curso de Bacharelado em Sistemas de Informação da USP. Como exemplo da aplicação bem sucedida dessa estratégia, apresentamos também um estudo de caso conduzido em 2009, em que parte das análises quantitativas aqui discutidas haviam sido aplicadas e fundamentaram a alteração do prérequisito entre as disciplinas Cálculo I e Cálculo II a partir de 2010. A disponibilidade de históricos acadêmicos antes e depois dessa alteração nos permitiu avaliar seu impacto nas taxas de reprovação em Cálculo II.

O restante deste artigo está organizado da seguinte maneira: a Seção 2 descreve os trabalhos correlatos; a Seção 3 apresenta o contexto no qual este estudo foi aplicado; a Seção 4 descreve a estratégia empregada; a Seção 5 descreve os resultados; por fim, a Seção 6 contém as conclusões e os trabalhos futuros.

\section{Trabalhos Correlatos}

A literatura correlata é vasta sobre criação ou aperfeiçoamento de estruturas curriculares adequadas para cursos da área de computação, bem como estratégias para diminuir as taxas de reprovação e evasão.

Há décadas associações internacionais trabalham para prover um currículo de referência, como é o caso da $\mathrm{ACM}^{1}$, $\mathrm{IEEE}^{2}$ e, no caso brasileiro, da $\mathrm{SBC}^{3}$.

\footnotetext{
${ }^{1}$ Association for Computing Machinery - http://www. acm.org/

${ }^{2}$ Institute of Electrical and Electronics Engineers - https: //www. ieee.org/

${ }^{3}$ Sociedade Brasileira de Computação - http://www. sbc . org.br
} 
Apenas para se ter uma ideia da amplitude desta discussão, Austing et al. (1977) apresentaram uma revisão da literatura na qual analisaram cerca de 200 trabalhos sobre o currículo de referência publicado pela ACM em 1968 (o primeiro publicado por essa associação). Os autores destacam que nas referências encontradas há diversos trabalhos sobre diferentes pontos de vista em relação às disciplinas recomendadas pelo currículo, desafios pedagógicos, sugestões de materiais complementares e discussões sobre algumas disciplinas específicas.

Em 1977, as duas principais associações internacionais da área apresentaram uma versão de seus relatórios sobre currículos de referência: "Curriculum Recommendations for the Undergraduate Program in Computer Science: A Report of the ACM Committee on Curriculum in Computer Science" da ACM e "A Curriculum in Computer Science and Engineering" da IEEE. Engel (1997) comparou os dois documentos e destacou a similaridade entre ambos, especialmente em relação às disciplinas de engenharia de software. Por outro lado, os dois documentos apresentavam certa diversidade quanto ao conteúdo relacionado a circuitos elétricos, circuitos lógicos, construção de computadores e teoria da computação.

A montagem destes currículos de referência era, tipicamente, realizada por pequenos comitês formados pelas associações da área. A partir de 1977, o comitê formado pela IEEE decidiu ampliar a participação da comunidade externa, enviando para membros selecionados da indústria, governo e academia um questionário sobre a versão em preparação de seu relatório. Sloan (1977) descreveu esta iniciativa, mostrando que as respostas dos questionários foram, em geral, bastante positivas. A autora destacou a necessidade de envolvimento maior da comunidade na criação desse tipo de relatório.

A última versão do currículo de referência preparado em conjunto pela ACM e IEEE data de 2013 [ACM and IEEE 2013]. Além do currículo para o curso de Ciência da Computação, estas entidades também apresentam currículos de referência para os cursos de Engenharia de Computação, Sistemas de Informação, Tecnologia da Informação e Engenharia de Software.

Apesar dos grandes esforços para oferecer uma currículo de referência completo, atual e que prepare os estudantes para o mercado de trabalho, é comum haver diferenças entre aquilo que é ensinado e aquilo que é procurado pelo empregador. Outlay e Krishnan (2010) propuseram uma metodologia para estudar essa diferença considerando o curso de Sistemas de Informação nos Estados Unidos. Os autores alegam que questões referentes ao aprendizado sobre legislação e lógica de processos costumam ser as mais destoantes entre o que é ensinado e o que é requerido pelos empregadores.

Frair et al. (1998) desenvolveram uma metodologia para avaliação de currículos com base na estratégia de processo de decisão chamada Processo Analítico Hierárquico. Os autores argumentam que este tipo de estratégia pode auxiliar no aperfeiçoamento ou mesmo criação de estruturas curriculares por meio da consulta a todas as partes afetadas (alunos, acadêmicos, empregadores, governos, etc). Esta consulta combinada com a estratégia proposta permite quantificar a importância de diferentes tópicos ou disciplinas de forma a guiar a criação de boas estruturas curriculares. Os autores informam que a metodologia ainda precisa ser melhor validada, mas que já os ajudou na criação de estruturas curriculares para o curso de Engenharia Industrial. 
Helfert e Duncan (2007) revisaram os trabalhos que discutiam dois currículos mais específicos: Information Systems e Business Informatics. Os autores destacaram as características interdisciplinares destes cursos, em particular no nível de pós-graduação e dos desafios de combinar habilidades de comunicação e gestão com aquelas oriundas das ciências exatas. É importante lembrar que nos Estados Unidos esses cursos de graduação tiveram origem na área de ciências humanas.

No contexto brasileiro, Nunes et al. (2015) especificaram uma metodologia para avaliar o gerenciamento acadêmico, que foi aplicada especificamente para cursos de engenharia e é baseada na análise de componentes principais. As análises apontaram para três grupos ortogonais de características, classificadas como: Formação Acadêmica, Formação Profissional e Gestão do Ensino Superior.

Também analisando dados brasileiros, Silva e Adeodato (2012) desenvolveram uma abordagem baseada em mineração de dados e regressão logística que permite identificar, ao final do segundo semestre do curso, alunos em situação de risco para evasão. $O$ estudo de caso analisou dados de seis cursos da Universidade Federal de Pernambuco e atingiu um desempenho de 0,84 para a medida área sobre a curva ROC. A identificação antecipada de alunos nessa situação é uma etapa importante para a realização de outras ações, como tutoria ou monitoria focadas nestes estudantes.

Marszalek et al. (2005) descrevem a estrutura e alguns resultados qualitativos e quantitativos do programa de mentoria que é aplicado na Universidade Tecnológica de Michigan desde 2002. Os autores apontam que estudantes que participaram deste programa tiveram resultados superiores aos demais em termos de taxa de egressos, especialmente nos grupos formados por minorias. Em termos de média geral, apesar de haver tendência de melhora entre os estudantes que participaram desse programa, os resultados não apresentaram boa significância estatística.

Satyanarayana et al. (2014) apresentaram resultados do programa de mentoria e tutoria aos alunos calouros na Escola de Tecnologia de Nova Iorque nos cursos de Sistemas de Informação e Engenharia de Computação. Neste programa, os alunos veteranos são os tutores dos calouros. Os autores avaliaram a taxa de aprovação dos alunos e concluíram que o programa de mentoria/tutoria conseguiu reduzir a evasão de $9 \%$ a $12 \%$.

Outra iniciativa comumente utilizada para aumentar o envolvimento dos alunos e diminuir a retenção é o envolvimento dos alunos em projetos científicos.

Dahlberg et al. (2008) apresentam um programa para envolver os estudantes de graduação em atividades de pesquisa, o que diminui a evasão e aumenta os interesses dos estudantes em fazer uma pós-graduação. As atividades de pesquisa são nas áreas de visualização, realidade virtual, robótica e jogos interativos, e os estudantes são imersos em grupos formados por outros estudantes de graduação, estudantes de pós-graduação e professores pesquisadores. Nesse trabalho, os autores apresentam os resultados dos primeiros dois anos de execução do programa e relatam o aumento das habilidades dos estudantes participantes e de seus desejos em desenvolver pesquisa científica.

Seguindo na mesma direção, Peckham et al. (2007) apresentam os resultados dos dois primeiros anos de aplicação do programa desenvolvido no curso de Ciência da Computação de Rhode Island para a redução da evasão e aumento da representatividade das mulheres no curso. O programa é baseado na apresentação dos alunos a pesquisas 
em computação gráfica, artes e novas mídias e combina práticas pedagógicas com mentorias, criando um ambiente no qual os estudantes sentem-se parte importante do grupo. De modo geral, a avaliação deste programa foi feita de forma qualitativa por meio de questionários respondidos pelos alunos. Os autores informam que os resultados superaram suas expectativas iniciais e elencam diversos aspectos que devem ser pensados para aqueles que desejem implementar um programa dessa natureza.

Thompson et al. (2014) também apresentam estratégias para diminuir a evasão por meio do envolvimento dos estudantes em atividades de pesquisa em cursos de computação, porém com o foco no recrutamento e permanência de mulheres nesses cursos. Inicialmente, os autores destacam seis características necessárias para que este tipo de iniciativa funcione: estratégia de recrutamento; permanência com pedagogia; permanência com currículo; permanência com apoio ao estudante; apoio institucional à iniciativa; e avaliação da iniciativa. Os autores descrevem diferentes estratégias adotadas por três universidades norte americanas destacando-se o recrutamento das mulheres ainda no ensino médio, o envolvimento em atividades de pesquisa, a integração das mulheres aos grupos existentes na universidade e a presença de tutores.

Crenshaw et al. (2008) apresentam os resultados de um estudo de dez meses relacionando a evasão com o senso de pertencimento dentro da Universidade de Illinois. Os autores concluem que os alunos de Ciência da Computação sentem-se muito isolados e que novas medidas precisam ser tomadas para aumentar o envolvimento e a diversidade. Adicionalmente, um cuidado especial precisa ser dado aos ingressantes. Os autores acreditam que por meio dessas medidas deverá haver redução da evasão.

Judson et al. (2015) avaliam a relação entre a evasão e o senso de pertencimento entre os estudantes de engenharia, analisando dados de 1998 a 2013. Os autores avaliaram diferentes estratégias utilizadas para o desenvolvimento do "senso de pertencimento", e mostram que as estratégias conseguiram aumentar a permanência (e reduzir a evasão) com boa efetividade. Entre as estratégias adotadas estão: desenvolvimento de atividades extracurriculares (como projetos científicos, escolas de verão para ingressantes e integração com associações profissionais); atualização da grade com duas novas disciplinas obrigatórias (uma sobre gerenciamento de tempo e outra sobre resolução de problemas e trabalho em equipe); e atividades de apoio estudantil, incluindo tutoria.

Barker et al. (2014) apresentam um estudo envolvendo cursos de computação de 14 universidades norte americanas. Os autores destacam o prejuízo mútuo no caso de abandono (evasão): o estudante investiu tempo e talvez dinheiro que não resultaram em sua graduação, e a universidade deixou de oferecer a vaga a outro estudante que poderia graduar-se. Assim, os autores investigaram quais são os principais fatores que levam um estudante a evadir-se, de forma que esta potencial evasão possa ser predita e, talvez, evitada. Dentre as principais conclusões sobre os dados analisados, os autores revelam que um dos principais fatores para evitar a evasão é o uso de atribuições significativas e relevantes aos estudantes, isto é, se o estudante não sentir que as disciplinas cursadas ou quaisquer outras atividades exigidas são relevantes para sua formação e/ou atuação profissional, ele facilmente poderá perder o interesse pela disciplina e, eventualmente, pelo curso. Outros fatores incluem boa interação/relacionamento entre cada estudante e o corpo docente e o desenvolvimento de atividades em grupo. Os autores destacam que o estudo revelou que a importância de cada fator varia bastante para cada um dos 
grupos estudados (por exemplo, medidas que são mais efetivas para diminuir a evasão de mulheres podem não ser as mais efetivas para reduzir a evasão de homens).

Monge et al. (2015) descrevem como a EngageCSEdu ${ }^{4}$, uma plataforma para o compartilhamento de materiais para auxiliar no engajamento dos alunos em disciplinas introdutórias de cursos de computação, pode ser utilizada para reduzir a evasão e aumentar o interesse dos estudantes e, em particular, dos grupos minoritários de estudantes desses cursos. Os autores destacam a importância de uma experiência positiva dos alunos nas disciplinas introdutórias de computação.

Digiampietri et al. (2013) apresentam duas atividades desenvolvidas no Bacharelado em Sistemas de Informação da USP para aumentar o envolvimento dos alunos no curso focadas em algoritmos e programação. Os autores destacam o campeonato anual de programação para calouros (BXCOMP) e as disciplinas optativas de Desafios de Programação I e II que, em cada aula, apresentam um ambiente semelhante a competições como a Maratona de Programação ${ }^{5}$. Além da elevada avaliação positiva entre os alunos participantes e do aumento de diferentes habilidades relatadas pelos alunos em questionários de autoavaliação, os autores relatam que estas iniciativas incentivaram os alunos a participarem de competições nacionais.

A contribuição original deste artigo é a proposta e avaliação de uma estratégia de análise quantitativa de revisão de pré-requisitos em disciplinas de um curso de Bacharelado em Sistemas de Informação. Conforme será apresentado na próxima seção, esta é apenas uma de várias iniciativas que vêm sendo tomadas desde o início do curso para seu aperfeiçoamento, atualização e redução de reprovação e evasão. A estratégia proposta pode ser considerada relevante devido à sua ampla aplicabilidade, seu baixo custo de implementação e a seu potencial de bons resultados, conforme será apresentado na seção de resultados.

\section{Contextualização}

O Bacharelado em Sistemas de Informação oferecido na Escola de Artes, Ciências e Humanidades da Universidade de São Paulo (USP) teve sua primeira turma de ingressantes em 2005 e recebe anualmente 180 alunos, divididos em três turmas de 60 alunos (uma matutina e duas noturnas).

Tanto a turma matutina quanto as noturnas possuem a mesma estrutura curricular ${ }^{6}$. O curso é composto por um total de 3.300 horas, incluindo 360 horas de estágio. Neste curso há 33 disciplinas específicas obrigatórias, 5 disciplinas optativas eletivas dentre um conjunto de 17 disciplinas do núcleo comum da unidade dividido em cinco áreas (também conhecido como Ciclo Básico) e mais 4 disciplinas optativas eletivas específicas do curso, dentro de uma gama de 25 disciplinas deste tipo. Todas as disciplinas oferecidas pelo curso são semestrais.

Cada disciplina pode ter zero ou mais disciplinas-requisito, que são disciplinas em que o aluno precisa superar um limite mínimo de aproveitamento para poder matricular-se

\footnotetext{
${ }^{4}$ https://www.engage-csedu.org/

$5_{\text {http: }} / /$ maratona.ime.usp.br/

${ }^{6}$ https://uspdigital.usp.br/jupiterweb/listarGradeCurricular?codcg=86\& codcur $=86200 \& \operatorname{codhab}=204 \& \mathrm{t} \mathrm{ipo}=\mathrm{N}$
} 
na primeira, o que constitui um pré-requisito. Há dois tipos de pré-requisitos de notas em vigor. O chamado pré-requisito forte implica que, para cursar uma dada disciplina, um aluno deverá ter sido aprovado na disciplina que é seu pré-requisito. Ser aprovado significa ter superado a frequência mínima e obtido nota maior ou igual a 5,0. Um exemplo deste pré-requisito ocorre com a disciplina Cálculo II: para cursá-la, o aluno deve ter sido aprovado na disciplina Cálculo I.

O outro tipo é chamado de pré-requisito fraco: para cursar uma dada disciplina o aluno deverá ter superado a frequência mínima e obtido nota maior ou igual a 3,0 na disciplina-requisito. Um exemplo deste pré-requisito ocorre com a disciplina Introdução a Análise de Algoritmos: para cursá-la, é necessário que o aluno tenha obtido presença mínima e nota maior ou igual a 3,0 em Introdução à Programação.

Atualmente, o curso conta com dezenas de pré-requisitos fracos e um único pré-requisito forte (a exigência de o aluno ter sido aprovado em Cálculo I para cursar Cálculo II).

Desde a criação do curso, várias medidas foram tomadas para seu aperfeiçoamento. A seguir, algumas destas medidas são brevemente apresentadas:

- diversas atualizações foram feitas na estrutura curricular ao longo dos últimos anos, com destaque para: troca de duas disciplinas do núcleo comum por uma disciplina obrigatória na área de sistemas distribuídos para a web, que era considerada uma lacuna da estrutura anterior; antecipação do oferecimento de disciplinas específicas do curso para o primeiro ano de graduação, visando a ampliar os conhecimentos específicos do aluno já no início do curso; revisão de diversos pré-requisitos realizada de maneira sistemática; e criação e oferecimento de novas optativas eletivas de acordo com o desejo dos alunos e a expertise dos professores.

- Duas alterações no exame vestibular foram realizadas para que o perfil dos ingressantes fosse mais compatível com os conhecimentos esperados pelas disciplinas iniciais do curso: a primeira foi a alteração nos pesos dados às provas do vestibular, aumentando o peso relativo das disciplinas de ciências exatas; a segunda foi a criação da carreira Computação da $\mathrm{FUVEST}^{7}$, em conjunto com os cursos de Ciência da Computação do Instituto de Matemática e Estatística (São Paulo) e do Instituto de Ciências Matemáticas e de Computação (São Carlos), o que ampliou o leque de candidatos (pois agora os alunos realizam um único exame com a perspectiva de poder cursar qualquer um desses três cursos) e aumentou as notas médias no vestibular dos alunos aprovados.

- Diversas atividades extracurriculares foram desenvolvidas no curso ao longo dos últimos anos. Destaca-se o uso de Coding Dojo para auxiliar os alunos calouros na aquisição e desenvolvimento de seus conhecimentos de programação; o campeonato anual de programação para calouros (BXCOMP); e a existência de um grupo de estudos em algoritmos que posteriormente evoluiu para o oferecimento de turmas das disciplinas Desafios de Programação I e II e para a participação dos alunos na Maratona de Programação, com uma equipe chegando até a final nacional da competição [Digiampietri et al. 2012].

\footnotetext{
${ }^{7}$ http://www.fuvest.br/
} 


\section{Metodologia}

A avaliação da estratégia proposta foi conduzida por meio de um estudo de caso, a partir de históricos acadêmicos de 1.361 alunos com matrículas ativas ou egressos do Bacharelado em Sistemas de Informação da USP, ingressantes entre 2005 e 2015 . Cada histórico acadêmico teve sua identidade apagada antes das análises, pois não fazia parte do trabalho a avaliação de alunos específicos.

Cada registro da base contém, essencialmente: número de matrícula do aluno no curso; código da disciplina; ano e semestre em que o aluno matriculou-se na disciplina; nota e frequência do aluno na disciplina.

Os desempenhos dos alunos foram confrontados par a par de disciplinas $\left(D_{a}, D_{d}\right)$, com o objetivo de identificar potencial dependência da disciplina $D_{d}$ em relação ao conteúdo anteriormente aprendido na disciplina $D_{a}$. Para isso, buscou-se responder à seguinte questão: a nota obtida por cada aluno em sua primeira matrícula na disciplina $D_{d}$ está associada ao desempenho anterior desse aluno na disciplina $D_{a}$ ?

Para essa análise, a partir da base de dados, obtivemos, para cada aluno que cursou a disciplina $D_{d}$, o par de notas $\left(N_{k, a}, N_{k, d}\right)$, em que $N_{k, d}$ denota a nota do $k$-ésimo aluno na disciplina $D_{d}$ na primeira vez em que a cursou, e $N_{k, a}$ denota a melhor nota obtida por esse mesmo aluno na disciplina $D_{a}$ antes de cursar $D_{d}$. Se o aluno não havia ainda cursado a disciplina $D_{a}$ antes de $D_{d}$, assumiu-se $N_{k, a}=0$.

As notas na disciplina $D_{a}$ foram categorizadas em três classes: de zero a três (três não incluso), correspondendo aos alunos que não cursaram $D_{a}$ ou tiveram desempenho muito baixo; de três a cinco (cinco não incluso), correspondendo aos alunos que tiveram desempenho insuficiente para aprovação, mas demonstraram ter aprendido parte dos conceitos da disciplina; e de cinco a dez, correspondendo aos alunos que mostraram ter assimilado o conteúdo da disciplina satisfatoriamente.

A principal métrica adotada foi o risco relativo de reprovação na disciplina $D_{d}$ em relação à disciplina $D_{a}$. O risco relativo ou razão de riscos é uma medida bastante utilizada em epidemiologia, e visa a comparar o risco de um evento adverso (doença, ferimento, morte, etc) em um grupo $A$ com o risco do evento em outro grupo $B$. Seu cálculo é feito dividindo-se a probabilidade do evento entre os indivíduos do grupo $A$ pela probabilidade do evento no grupo $B$ [CDC 2006].

Neste estudo, adotou-se o risco relativo para comparar as taxas de reprovações da disciplina $D_{d}$ nos três grupos de alunos definidos para a disciplina $D_{a}$. Por exemplo, o risco relativo de reprovação na disciplina $D_{d}$ de um aluno que não tenha atingido o prérequisito fraco na disciplina $D_{a}$ (ou seja, não cursou ou tirou nota inferior a 3,0 em $D_{a}$ ) em relação a um aluno que foi aprovado em $D_{a}$ pode ser estimado pela equação 1 .

$$
R R_{03 / A p r}=\frac{\% \operatorname{Repr} D_{d} \mid 03 D_{a}}{\% \operatorname{Repr} D_{d} \mid A p r D_{a}}
$$

sendo $\% \operatorname{Repr} D_{d} \mid 03 D_{a}$ a porcentagem dos alunos reprovados na disciplina $D_{d}$ dentre os que não cursaram ou obtiveram notas inferiores a 3,0 na disciplina $D_{a} \mathrm{e}$ $\% \operatorname{Repr} D_{d} \mid A p r D_{a}$ é a porcentagem dos alunos reprovados na disciplina $D_{d}$ dentre os que passaram na disciplina $D_{a}$. 
Assim pode-se estimar, por exemplo, que um aluno que não tenha cursado ou tenha obtido nota inferior a 3,0 em $D_{a}$ tem o dobro de chance de reprovar na disciplina $D_{d}$ em relação àquele que foi aprovado em $D_{a}$. Tal observação poderia indicar a necessidade de revisão de pré-requisitos entre as disciplinas $D_{a}$ e $D_{d}$.

A escolha desse indicador de associação foi motivada por seu amplo uso em análise de risco e por sua simplicidade de cálculo e de interpretação.

Ao todo foram analisados 1.260 pares de disciplinas e alguns filtros foram passados para excluir subpopulações muito esparsas, especialmente aquelas decorrentes de disciplinas cursadas por quantidades muito pequenas de alunos. Para o estudo, foram consideradas exclusivamente as disciplinas específicas do curso (obrigatórias ou optativas) nas quais ao menos $15 \%$ dos alunos do estudo tivessem se matriculado. Adicionalmente, somente foram considerados os pares de disciplinas $\left(D_{a}, D_{d}\right)$ em que a taxa de reprovação em $D_{d}$ fosse superior a $15 \%$ e a taxa de reprovação em $D_{a}$ estivesse entre $25 \%$ e $75 \%$ (inclusive). Esse critério visava a identificar pares em que $D_{d}$ tivesse taxas moderadas ou altas de reprovação, de forma que as subpopulações de alunos em relação a $D_{a}$ (reprovados ou aprovados) tivessem uma representação mínima de $25 \%$. Não houve qualquer restrição em relação aos semestres de oferecimento. Os pares $\left(D_{a}, D_{d}\right)$ foram ranqueados em ordem decrescente de $R R_{03 / A p r}$.

\section{Resultados}

Os resultados foram analisados considerando três aspectos que serão apresentados e discutidos ao longo desta seção:

1. Dentre os alunos reprovados na disciplina potencialmente dependente $D_{d}$, qual havia sido seu desempenho na disciplina prévia $D_{a}$ ?

2. Dado o desempenho dos alunos na disciplina $D_{a}$, qual seu risco de reprovação posteriormente na disciplina $D_{d}$ ?

3. Por fim, será apresentado o risco relativo de reprovação dos alunos na disciplina $D_{d}$ entre os seguintes grupos de alunos:

- Alunos com notas inferiores a 3,0 $\times$ alunos com notas entre 3,0 e 5,0 em $D_{a}$;

- Alunos com notas inferiores a 3,0 $\times$ alunos aprovados em $D_{a}$;

- Alunos com notas entre 3,0 e 5,0 $\times$ alunos aprovados em $D_{a}$;

A Tabela 1 apresenta as siglas utilizadas nas figuras desta seção e seus significados.

A fim de entender-se o comportamento geral dos desempenhos considerando o desempenho em um par de disciplinas, diversos cálculos foram realizados. O primeiro visa a entender quais notas os alunos haviam obtido anteriormente na disciplina $D_{a}$ sendo que, posteriormente, eles reprovaram na disciplina $D_{d}$. As Figuras 1, 2 e 3 ilustram esse comportamento. Para todos os alunos que reprovaram na disciplina $D_{d}$, cada uma das figuras representa um desempenho diferente na disciplina $D_{a}$, isto é, cada figura apresenta a distribuição da probabilidade condicional dos alunos que reprovaram na disciplina $D_{d}$ terem tido um dado desempenho na disciplina $D_{a}$.

A Figura 1 contém os dados dos alunos que não haviam cursado ou haviam obtido notas inferiores a 3,0 na disciplina $D_{a}$ quando reprovaram na disciplina $D_{d}$; a Figura 2 
Tabela 1. Siglas utilizadas e seus significados

\begin{tabular}{|l|l|}
\hline Sigla & Legenda \\
\hline$\% 03 D_{a} \mid \operatorname{Repr} D_{d}$ & $\begin{array}{l}\text { Porcentagem dos alunos que não cursaram ou obtiveram notas inferiores a 3,0 na } \\
\text { disciplina } D_{a} \text { dentre os que foram reprovados na disciplina } D_{d}\end{array}$ \\
\hline$\% 35 D_{a} \mid \operatorname{Repr} D_{d}$ & $\begin{array}{l}\text { Porcentagem dos alunos com notas entre 3,0 e 5,0 (cinco não incluso) na disci- } \\
\text { plina } D_{a} \text { dentre os que foram reprovados na disciplina } D_{d}\end{array}$ \\
\hline$\%$ Apr $D_{a} \mid \operatorname{Repr} D_{d}$ & $\begin{array}{l}\text { Porcentagem dos alunos que passaram na disciplina } D_{a} \text { dentre os que foram re- } \\
\text { provados na disciplina } D_{d}\end{array}$ \\
\hline$\% \operatorname{Repr} D_{d} \mid 03 D_{a}$ & $\begin{array}{l}\text { Porcentagem dos alunos reprovados na disciplina } D_{d} \text { dentre os que não cursaram } \\
\text { ou obtiveram notas inferiores a 3,0 na disciplina } D_{a}\end{array}$ \\
\hline$\% \operatorname{Repr} D_{d} \mid 35 D_{a}$ & $\begin{array}{l}\text { Porcentagem dos alunos reprovados na disciplina } D_{d} \text { dentre os que obtiveram } \\
\text { notas entre 3,0 e 5,0 na disciplina } D_{a}\end{array}$ \\
\hline$\% \operatorname{Repr} D_{d} \mid$ Apr $D_{a}$ & $\begin{array}{l}\text { Porcentagem dos alunos reprovados na disciplina } D_{d} \text { dentre os que passaram na } \\
\text { disciplina } D_{a}\end{array}$ \\
\hline
\end{tabular}

contém os dados dos alunos com notas entre 3,0 e 5,0 na disciplina $D_{a}$; a Figura 3 apresenta os dados dos alunos que haviam sido aprovados na disciplina $D_{a}$ antes de terem reprovado na disciplina $D_{d}$.

Para cada uma destas figuras, cada barra corresponde à porcentagem de pares de disciplinas que se enquadraram no respectivo intervalo. Por exemplo, dentre os 1.260 pares de disciplinas analisados, em cerca de $11 \%$ dos pares os alunos que não haviam cursado ou haviam obtido notas inferiores a 3,0 na disciplina $D_{a}$ corresponderam a de $0 \%$ a $5 \%$ do total de reprovações na disciplina $D_{d}$ (ver primeira barra da Figura 1); ou seja, ao menos $95 \%$ das reprovações na disciplina $D_{d}$ ocorreram entre alunos com notas maiores do que 3,0 na disciplina $D_{a}$ (isto pode ser explicado, por exemplo, pela presença de prérequisitos na disciplina $D_{d}$ ). No outro extremo da Figura 1 observamos que, em cerca de $35 \%$ dos pares de disciplinas analisados, mais de $95 \%$ das reprovações na disciplina $D_{d}$ ocorreram entre os alunos que não haviam cursado ou haviam obtido notas inferiores a 3,0 na disciplina $D_{a}$.

A distribuição de probabilidades exibida na Figura 2 apresenta um comportamento bastante diferente do apresentado pela Figura 1. Na Figura 2 observa-se uma grande concentração de ocorrências em intervalos de probabilidade baixos. Por exemplo, a porcentagem de alunos reprovados na disciplina $D_{d}$ e que tinham obtido notas entre 3,0 e 5,0 na disciplina $D_{a}$ foi de $0 \%$ a $5 \%$ em quase $70 \%$ dos pares de disciplinas analisados.

A Figura 3 apresenta a distribuição das probabilidades dos pares de disciplinas considerando a probabilidade condicional de aprovação na disciplina $D_{a}$ dentre os alunos posteriormente reprovados na disciplina $D_{d}$. Observa-se que, para cerca de $35 \%$ dos pares de disciplinas, os alunos que haviam sido aprovados na disciplina $D_{a}$ e reprovaram na disciplina $D_{d}$ correspondem a apenas de $0 \%$ a $5 \%$ do total de reprovados na disciplina $D_{d}$. Ao se somar os valores das três primeiras barras desta figura, verifica-se que para metade dos pares de disciplinas analisados, dentre os reprovados na disciplina $D_{d}$, menos de $15 \%$ deles haviam sido aprovados na disciplina $D_{a}$.

No outro extremo da Figura 3 observa-se que em cerca de $7 \%$ dos pares de disciplinas analisados, dentre os reprovados na disciplina $D_{d}, 95 \%$ ou mais dos alunos haviam sido aprovados na disciplina $D_{a}$. Isto poderia indicar que, para estes casos, cursar ou não a disciplina $D_{a}$ não interfere diretamente no desempenho da disciplina $D_{d}$. 
As três figuras apresentadas ilustram o comportamento geral do desempenho dos alunos na disciplina $D_{a}$, dado que reprovaram na disciplina $D_{d}$. Embora essa análise seja útil para se ter uma ideia do perfil dos alunos que reprovaram na disciplina $D_{d}$, há diversas informações adicionais que precisam ser consideradas. Por exemplo, se a disciplina $D_{a}$ for pré-requisito fraco da disciplina $D_{d}$, então não é esperada a existência de vários alunos reprovados na disciplina $D_{d}$ que não tenham cursado ou que tenham obtido notas inferiores a 3,0 na disciplina $D_{a}$; isto, de certa forma, explica os valores das primeiras barras da Figura 1, que são mais altos do que os valores subsequentes.

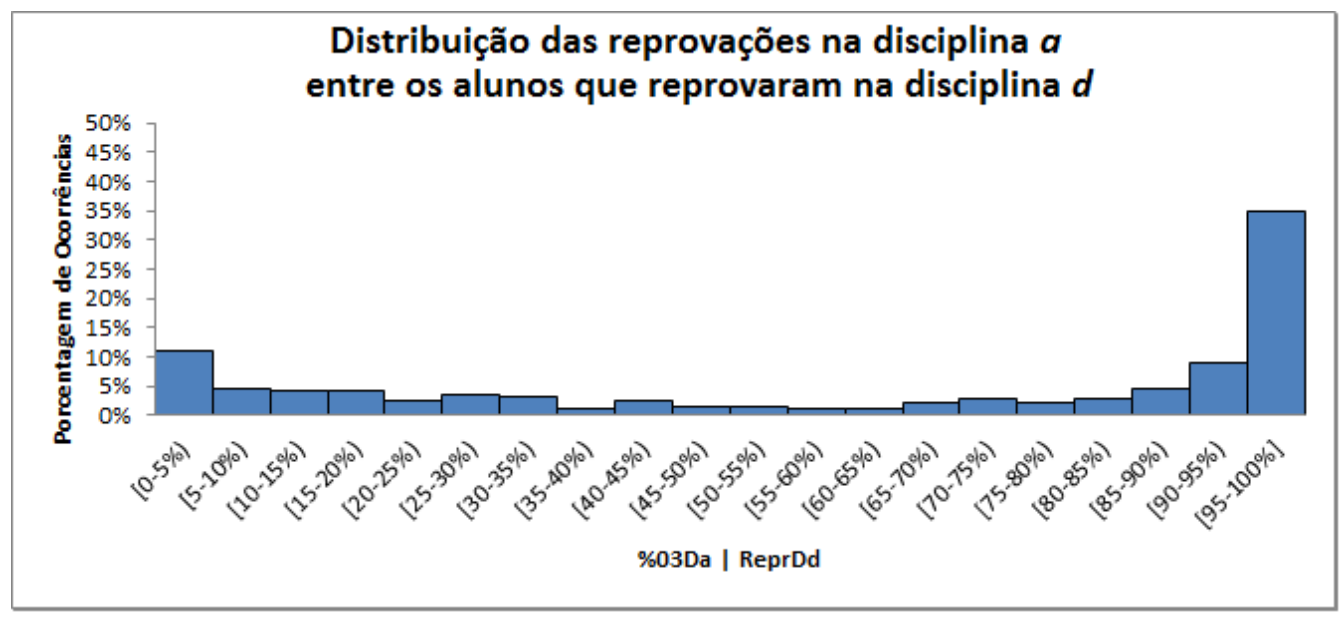

Figura 1. Distribuição das ocorrências nas quais os alunos que reprovaram na disciplina $D_{d}$ haviam obtido notas inferiores a 3,0 na disciplina $D_{a}$ ou não haviam cursado esta disciplina

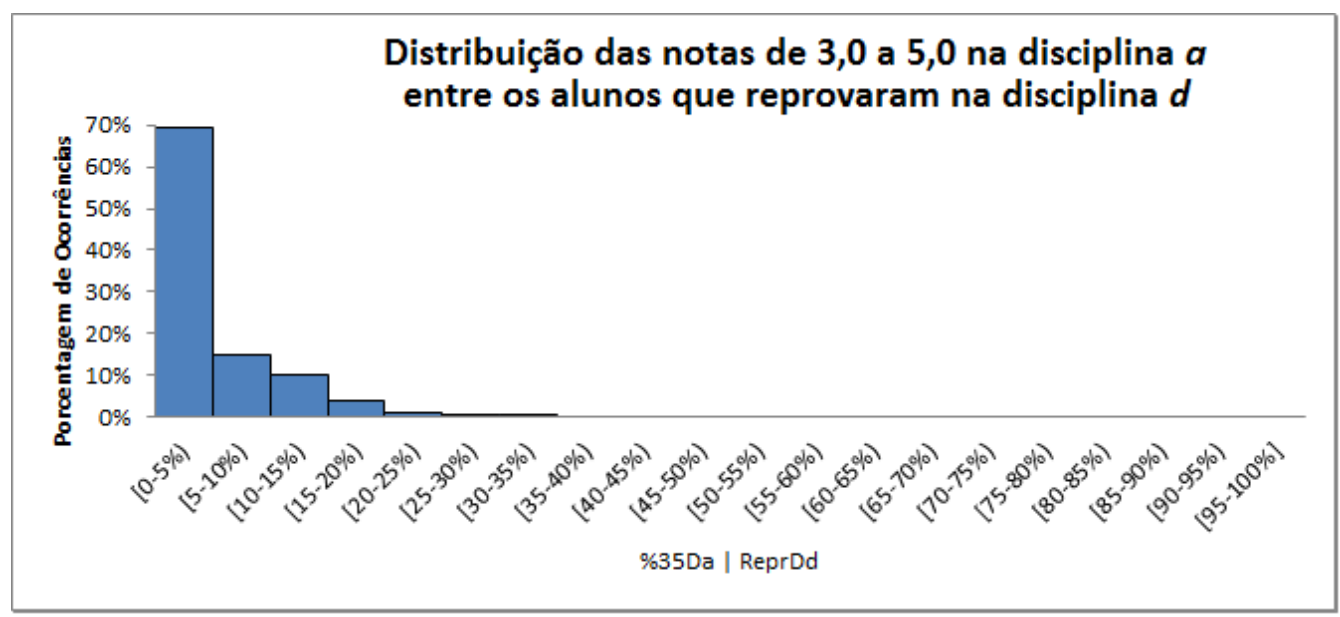

Figura 2. Distribuição das ocorrências nas quais os alunos que reprovaram na disciplina $D_{d}$ haviam obtido notas entre 3,0 a 5,0 na disciplina $D_{a}$

Estas três figuras apresentaram informações relativas a: dado que o aluno foi reprovado na disciplina $D_{d}$, qual foi sua situação na disciplina $D_{a}$ ? Outra informação importante neste contexto é: dado que o aluno apresentou um certo desempenho na disciplina $D_{a}$, qual será a implicação na disciplina $D_{d}$ ? Uma diferença fundamental entre estas duas análises é que na primeira não se tem informação sobre o total de alunos que apresentaram um dado comportamento na disciplina $D_{a}$, mas apenas daqueles que também 


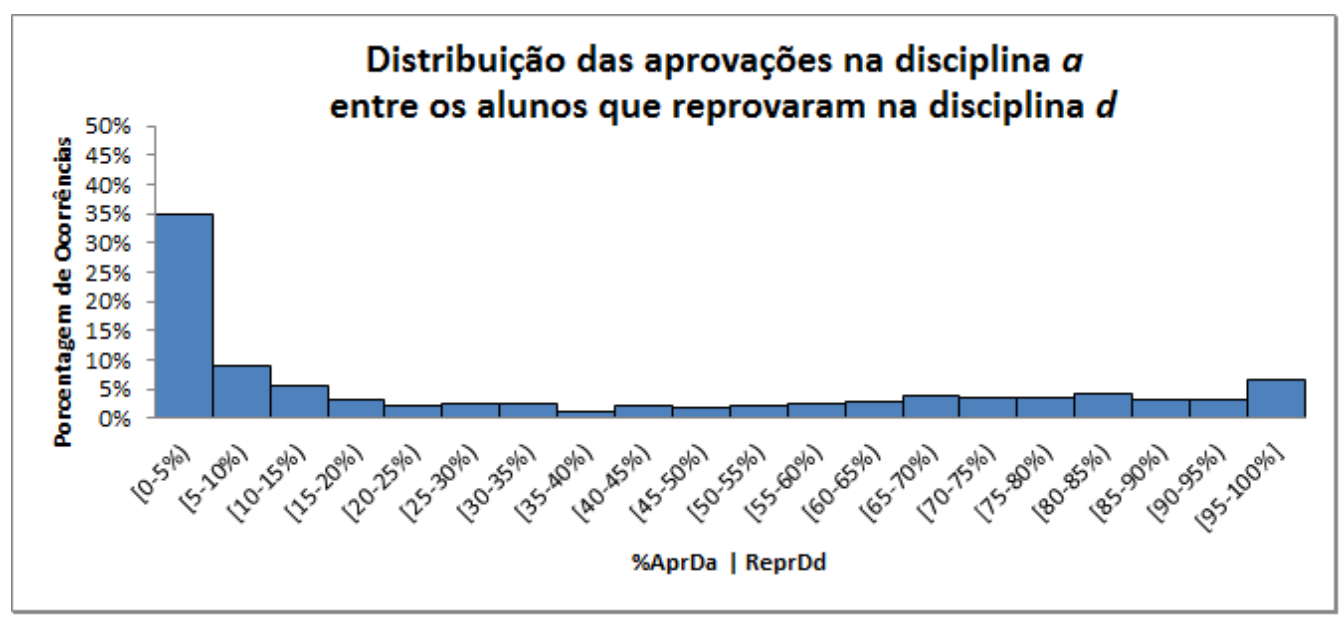

Figura 3. Distribuição das ocorrências nas quais os alunos que reprovaram na disciplina $D_{d}$ haviam sido aprovados na disciplina $D_{a}$

reprovaram na disciplina $D_{d}$. Por exemplo, se $90 \%$ dos alunos que tiraram menos que 3,0 na disciplina $D_{a}$ ou não a cursaram foram aprovados na disciplina $D_{d}$, mas, apesar disso, $75 \%$ dos que reprovaram na disciplina $D_{d}$ não haviam cursado ou haviam obtido notas inferiores a 3,0 na disciplina $D_{a}$, esta última informação poderia sugerir que é importante a criação de um pré-requisito fraco na disciplina $D_{d}$ em relação à disciplina $D_{a}$. Isto provavelmente seria um equívoco, já que $90 \%$ dos alunos que obtiveram notas menores que 3,0 em $D_{a}$ (ou não a cursaram) passaram na disciplina $D_{d}$.

Assim, dado o desempenho na disciplina $D_{a}$, as Figuras 4, 5 e 6 apresentam a distribuição das reprovações na disciplina $D_{d}$.

A Figura 4 mostra que, para a maioria dos pares de disciplinas estudados, o baixo desempenho na disciplina $D_{a}$ (ou o fato de o aluno ainda não a ter cursado) implica em uma taxa de reprovação de $15 \%$ a $30 \%$ na disciplina $D_{d}$. São mais relevantes para este estudo os casos no extremo da figura em que o fato de o aluno ter obtido nota inferior a 3,0 ou não ter cursado a disciplina $D_{a}$ acarreta em taxas de reprovação na disciplina $D_{d}$ maiores ou iguais a $50 \%$ (há 57 pares de disciplinas que se enquadram nesta situação).

$\mathrm{Na}$ Figura 5 são apresentados os dados referentes aos alunos que obtiveram notas entre 3,0 e 5,0 na disciplina $D_{a}$ e reprovaram na disciplina $D_{d}$. Observa-se que, para mais de $45 \%$ dos pares de disciplinas, a taxa de reprovação na disciplina $D_{d}$ ficou entre $0 \%$ e $5 \%$. Em $12,5 \%$ dos pares de disciplinas estudados, a taxa de reprovação na disciplina $D_{d}$ entre os alunos que tiraram de 3,0 a 5,0 na disciplina $D_{a}$ foi maior ou igual a $50 \%$.

A Figura 6 contém os dados sobre as reprovações na disciplina $D_{d}$, dado que o aluno foi aprovado na disciplina $D_{a}$. Para mais de $80 \%$ dos pares de disciplinas estudados a taxa de reprovação na disciplina $D_{d}$ foi menor do que $25 \%$.

As medidas discutidas até o momento caracterizam o perfil do aluno que reprovou na disciplina $D_{d}$ e, também, o risco de o aluno ser reprovado na disciplina $D_{d}$ de acordo com seu desempenho na disciplina $D_{a}$. Uma forma mais eficaz de analisar o risco de um aluno reprovar na disciplina $D_{d}$ dado seu desempenho na disciplina $D_{a}$ é de forma relativa, comparando o risco de o aluno ser reprovado na disciplina $D_{d}$ dado seu desempe- 
nho na disciplina $D_{a}$ em relação a este risco para alunos que obtiveram um desempenho melhor na disciplina $D_{a}$.

Três tipos de riscos relativos de reprovação na disciplina $D_{d}$ em relação à situação da disciplina $D_{a}$ foram analisados: o risco relativo entre cursar a disciplina $D_{d}$ sem ter obtido ao menos 3,0 na disciplina $D_{a}$ em relação a ter cursado a disciplina $D_{a}$ e ter obtido nota entre 3,0 e 5,0 (RiscoRel 03/35); o risco relativo de cursar a disciplina $D_{d}$ sem ter obtido ao menos 3,0 na disciplina $D_{a}$ em relação aos alunos aprovados na disciplina $D_{a}$ (RiscoRel 03/Apr); e o risco relativo de se ter obtido de 3,0 a 5,0 na disciplina $D_{a} \mathrm{em}$ relação aos alunos que foram aprovados na disciplina $D_{a}$ (RiscoRel 35/Apr).

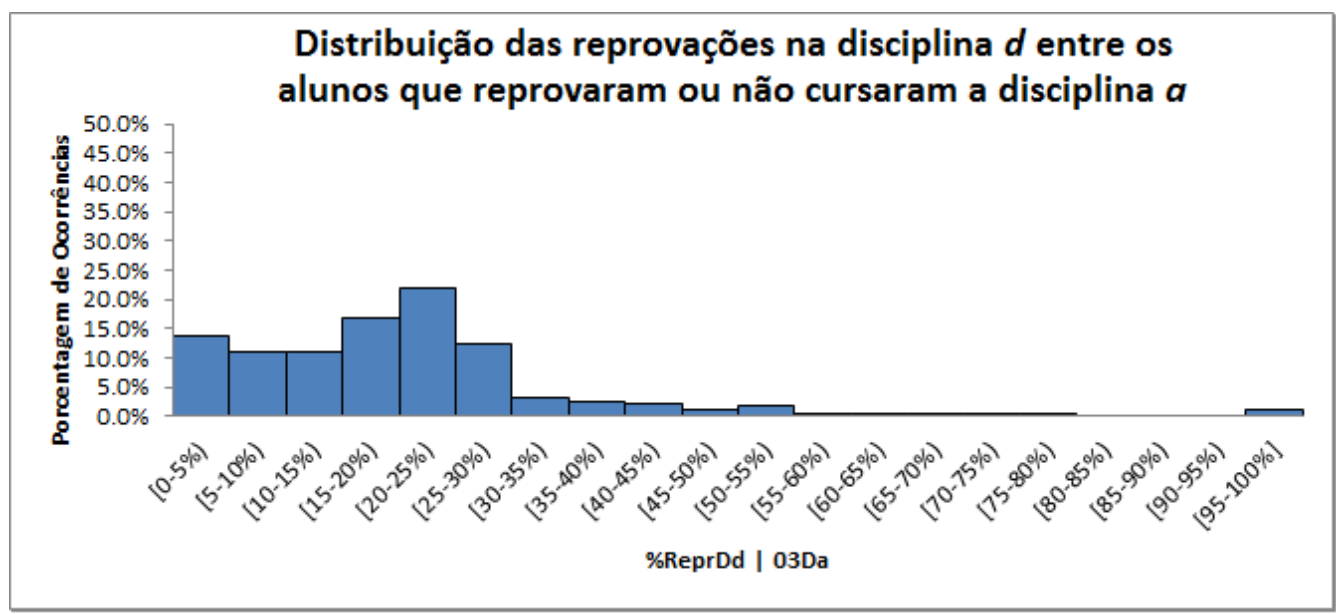

Figura 4. Distribuição das ocorrências nas quais os alunos que haviam tirado menos que 3,0 ou não haviam cursado a disciplina $D_{a}$ reprovaram na disciplina $D_{d}$

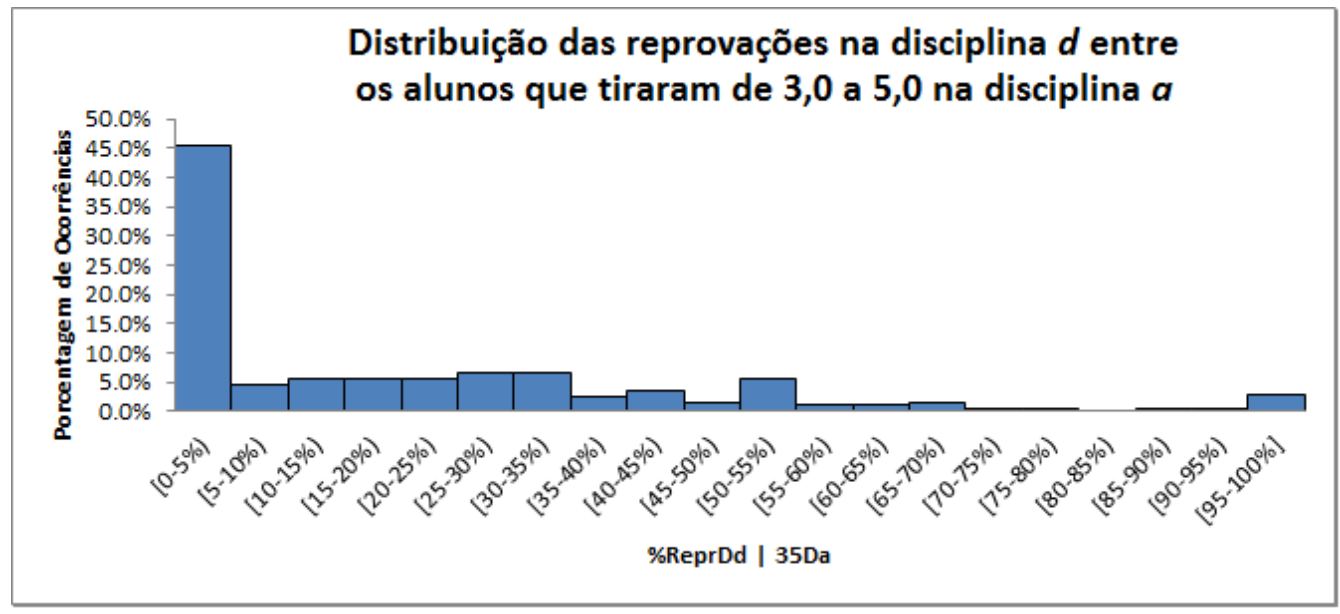

Figura 5. Distribuição das ocorrências nas quais os alunos que haviam tirado de 3,0 a 5,0 na disciplina $D_{a}$ reprovaram na disciplina $D_{d}$

A Tabela 2 contém os doze maiores riscos relativos, em ordem decrescente em relação a RiscoRel 03/Apr, isto é, o risco relativo de reprovação em $D_{d}$ dos alunos que não cursaram ou que obtiveram notas inferiores a 3,0 em $D_{a}$ em relação aos alunos que foram aprovados em $D_{a}$. 


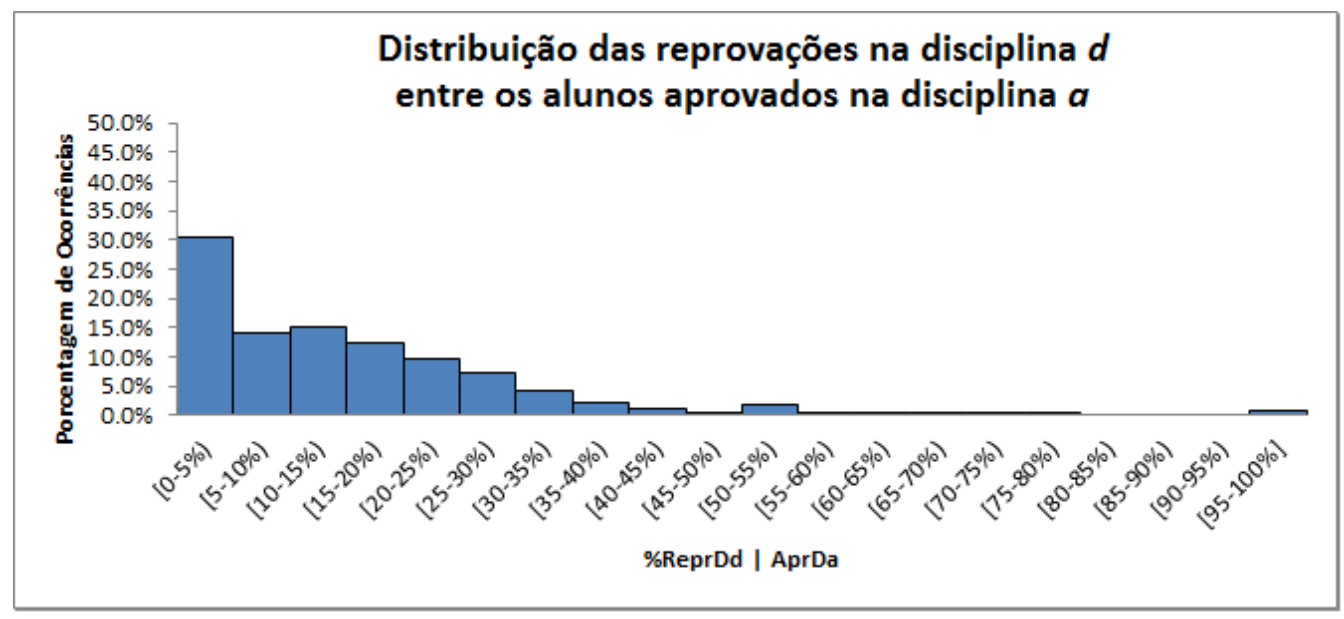

Figura 6. Distribuição das ocorrências nas quais os alunos que haviam sido aprovados na disciplina $D_{a}$ reprovaram na disciplina $D_{d}$

Tabela 2. Doze maiores riscos relativos 03/Apr entre as disciplinas obrigatórias do curso

\begin{tabular}{|l|l|c|c|c|}
\hline do CurSo & Risciplinas $D_{d}$ & $\begin{array}{c}\text { RiscoRel } \\
03 / 35\end{array}$ & $\begin{array}{c}\text { RiscoRel } \\
03 / \text { Apr }\end{array}$ & $35 /$ Apr \\
\hline Disciplina $D_{a}$ & Matemática Discreta I & 1,56 & 5,79 & 3,7 \\
\hline Cálculo II & Organização de Computadores Digitais & 1,22 & 4,65 & 3,82 \\
\hline Introdução à Estatística & 1,28 & 4,56 & 3,56 \\
\hline Introdução à Análise de Algoritmos & Matemática Discreta I & 2,15 & 4,09 & 1,9 \\
\hline Computação Orientada a Objetos & Organização de Computadores Digitais & 1,73 & 3,93 & 2,27 \\
\hline Algoritmos e Estruturas de Dados I & Organizção de Computadores Digitais & 1,19 & 3,46 & 2,92 \\
\hline Matrizes, Vetores e Geometria Analítica & Organizção de Computadores Digitais & 1,58 & 3,18 & 2,02 \\
\hline Introdução à Estatística & Bancos de Dados & 1,32 & 3,18 & 2,41 \\
\hline Algoritmos e Estruturas de Dados II & Arquitetura de Computadores & 0,98 & 2,97 & 3,02 \\
\hline Introdução à Análise de Algoritmos & Algoritmos e Estruturas de Dados I & 0,85 & 2,53 & 2,97 \\
\hline Fundamentos de Sistemas de Informação & Cálculo II & 0,86 & 2,37 & 2,75 \\
\hline Introdução à Estatística & Algoritmos e Estruturas de Dados II & 1,13 & 2,35 & 2,08 \\
\hline Cálculo II & Algoritmos e Estruturas de Dados I & & \\
\hline
\end{tabular}

Destacam-se três situações a partir da Tabela 2. A primeira é o maior risco 03/Apr apresentado na primeira linha de dados da tabela, indicando que os alunos que não cursaram Cálculo II ou obtiveram nota inferior a 3,0 têm 5,79 mais chances de reprovar em Matemática Discreta do que os alunos que foram aprovados em Cálculo II.

A segunda situação é o valor 2,15 para o risco relativo 03/35 tendo como disciplina $D_{a}$ Computação Orientada a Objetos e como disciplina $D_{d}$ Organização de Computadores Digitais. Esse valor indica que os alunos que cursam Organização de Computadores Digitais não tendo cursado Computação Orientada a Objetos ou obtendo uma média inferior a 3,0 têm mais que o dobro de chance de reprovar em Organização de Computadores Digitais em relação àqueles que tiraram entre 3,0 e 5,0 em Computação Orientada a Objetos.

A terceira situação de destaque é que a disciplina Organização de Computadores Digitais apareceu quatro vezes como disciplina $D_{d}$ na Tabela 2, o que pode ser um forte indício da necessidade da criação de um pré-requisito para esta disciplina.

Os resultados apresentados acima são baseados em históricos acadêmicos de 2005 a 2015, e são focados na identificação de eventuais associações entre pares de disciplinas que não fazem parte dos pré-requisitos atuais no Bacharelado em Sistemas de Informação 
da USP. Não obstante, parte das estratégias de análise aqui apresentadas já haviam sido aplicadas em 2009, a fim de identificar a associação entre as disciplinas Cálculo I e Cálculo II. Este caso é comentado brevemente abaixo, e representa um exemplo bem sucedido da aplicação da estratégia proposta e corrobora a hipótese deste trabalho.

Até 2009, a disciplina Cálculo II, que tinha uma taxa de reprovação média de 30,4\%, possuía Cálculo I como pré-requisito fraco. Analisando-se os históricos acadêmicos dos alunos de 2005 a 2009, identificou-se que o risco de reprovação em Cálculo II entre aqueles que tinham obtido notas entre 3,0 e 5,0 em Cálculo I era 2,86 vezes maior do que o dos alunos que tivessem sido aprovados em Cálculo I. A constatação dessa maior chance de reprovação fez com que, em 2010, o pré-requisito fosse alterado para forte. A partir dessa mudança a taxa total de reprovação em Cálculo II no período de 2010 a 2015 teve uma redução de 52,6\%, passando para 14,4\%. É interessante notar que essa expressiva redução é consistente com o risco relativo calculado, e parece confirmar a hipótese de que a obrigatoriedade de o aluno obter uma base mais sólida em Cálculo I antes de se matricular em Cálculo II diminui seu risco de reprovação nesta disciplina.

\section{Conclusões e Trabalhos Futuros}

Existem diversos desafios para manter a estrutura curricular atualizada e adequada aos alunos, prendendo o interesse destes, mantendo-os motivados e preparando-os para o mercado de trabalho.

A reprovação sucessiva em disciplinas pode ser um gatilho para que alunos evadam de um curso de graduação. Uma causa potencial de reprovação em uma disciplina é a falta de preparo adequado do aluno, que poderia ser propiciado nas disciplinas previamente cursadas. Assim, a alteração de pré-requisitos pode ser utilizada para garantir uma melhor base de conhecimentos ao aluno antes deste matricular-se em uma dada disciplina, diminuindo assim suas chances de reprovação na mesma. Todavia, a introdução de prérequisitos tem sido usualmente feita exclusivamente com base nas análises de conteúdos entre disciplinas, sem o suporte de análise de associação entre disciplinas com base no desempenho dos estudantes.

A questão de pesquisa deste trabalho é o papel dos pré-requisitos como mecanismos para melhorar o aproveitamento e o desempenho acadêmico dos estudantes. Mais especificamente, a hipótese aqui estabelecida é de que o desempenho médio de um aluno em algumas disciplinas de níveis intermediários e avançados (oferecidas a partir do $2^{\circ}$ semestre) depende do desempenho desse aluno em disciplinas cursadas anteriormente.

Este artigo apresentou uma estratégia de análise quantitativa, conduzida no Bacharelado em Sistemas de Informação da USP, para o cálculo do risco relativo de se cursar uma disciplina dado o desempenho dos alunos em disciplinas anteriores. Essa métrica é útil para a identificação de associações entre desempenhos em diferentes disciplinas e pode servir de base para a atualização de pré-requisitos. Dois pré-requisitos potenciais identificados seriam:

- Cálculo II como pré-requisito para Matemática Discreta;

- Computação Orientada a Objetos como pré-requisito para Organização de Computadores Digitais.

Pretende-se submeter os resultados obtidos neste estudo à avaliação dos pares e da Comissão Coordenadora do Curso a fim de ponderar a necessidade e a viabilidade de 
implantação desses pré-requisitos potenciais - que implicariam no deslocamentos das disciplinas na grade curricular, já que as disciplinas envolvidas são oferecidas atualmente nos mesmos semestres.

Parte da estratégia aqui apresentada foi utilizada anteriormente, tendo auxiliado na decisão de tornar Cálculo I um pré-requisito forte para Cálculo II a partir de 2010 (ou seja, para se matricular em Cálculo II, o aluno precisa ter sido aprovado em Cálculo I). A partir dessa alteração, a taxa média anual de reprovação em Cálculo II teve redução superior a $50 \%$.

A estratégia apresentada possui algumas limitações a serem exploradas e mitigadas no futuro. A primeira limitação é que o indicador de associação adotado neste estudo não permite afirmar que o risco de reprovação deve-se somente à dependência entre as disciplinas e não a outros fatores, tais como tendências individuais dos alunos de obter melhores ou piores notas independentemente da disciplina, ou diferenças de aptidões individuais nas diferentes áreas disciplinares - que podem induzir associações numéricas entre disciplinas sem necessariamente implicar em associações causais. Trabalhos futuros deverão explorar medidas de desempenho normalizadas, que busquem atenuar a influência desses fatores.

Implementações futuras deverão também incluir intervalos estatísticos para os valores dos indicadores, para a obtenção de resultados mais robustos e menos suscetíveis à variabilidade induzida por subgrupos muito pequenos. Tal situação pode ocorrer, por exemplo, com disciplinas com frequências muito baixas de reprovação ou com frequências muito baixas de notas inferiores a 3,0. Técnicas de bootstrap são uma alternativa simples e robusta para essa finalidade [Efron and Tibshirani 1993].

Adicionalmente, pretende-se analisar todas as mudanças de pré-requisitos ocorridas desde a criação do curso, a fim de avaliar os diferentes efeitos nas taxas de reprovação nas disciplinas envolvidas.

\section{Agradecimentos}

Os autores agradecem à Pró-Reitoria de Graduação da Universidade de São Paulo, à FAPESP (Processos 2010/50797-4, 2012/04788-9, 2013/07375-0) e ao CNPq (Processos 477246/2013-3 e 306046/2013-0).

\section{Referências}

[ACM and IEEE 2013] ACM and IEEE (2013). Computer Science Curricula 2013: Curriculum Guidelines for Undergraduate Degree Programs in Computer Science. ACM, New York, NY, USA. 999133.

[Austing et al. 1977] Austing, R. H., Barnes, B. H., and Engel, G. L. (1977). A survey of the literature in computer science education since curriculum '68. Commun. ACM, 20(1):13-21.

[Barker et al. 2014] Barker, L., Hovey, C., and Thompson, L. (2014). Results of a largescale, multi-institutional study of undergraduate retention in computing. In Frontiers in Education Conference (FIE), 2014 IEEE, pages 1-8. 
[Barros da Silva and Leitao Adeodato 2012] Barros da Silva, H. and Leitao Adeodato, P. (2012). A data mining approach for preventing undergraduate students retention. In Neural Networks (IJCNN), The 2012 International Joint Conference on, pages 1-8.

[CDC 2006] CDC (2006). Principles of Epidemiology in Public Health Practice. Center for Disease Control and Prevention, Atlanta, GA, USA, 3rd edition.

[Crenshaw et al. 2008] Crenshaw, T. L., Chambers, E. W., and Metcalf, H. (2008). A case study of retention practices at the university of illinois at urbana-champaign. In Proceedings of the 39th SIGCSE Technical Symposium on Computer Science Education, SIGCSE '08, pages 412-416, New York, NY, USA. ACM.

[Dahlberg et al. 2008] Dahlberg, T., Barnes, T., Rorrer, A., Powell, E., and Cairco, L. (2008). Improving retention and graduate recruitment through immersive research experiences for undergraduates. In Proceedings of the 39th SIGCSE Technical Symposium on Computer Science Education, SIGCSE '08, pages 466-470, New York, NY, USA. ACM.

[Digiampietri et al. 2012] Digiampietri, L., Peres, S., Nakano, F., Roman, N., da Silva, B., and Teodoro, B. (2012). Complementando o aprendizado em programação: Experiências no curso de sistemas de informação da usp. In SBSI 2012 - Trilhas Técnicas (Technical Tracks).

[Digiampietri et al. 2013] Digiampietri, L. A., Peres, S. M., Nakano, F., Roman, N., Wagner, P. K., Silva, B. B. C., Teodoro, B., Silva-Junior, D. F. P., Pereira, G. V. A., Borges, G. O., Pereira, G. R., Santos, M. V., Baklizky, M., and Barros, V. A. (2013). Complementando o aprendizado em programação: Revisitando experiências no curso de sistemas de informação da usp. iSys: Revista Brasileira de Sistemas de Informação, 6:5-29.

[Efron and Tibshirani 1993] Efron, B. and Tibshirani, R. (1993). An Introduction to the Bootstrap. Chapman and Hall, New York, USA.

[Engel 1977] Engel, G. (1977). A comparison of the acmic3s and the ieee/cse model curriculum subcommittee recommendations. Computer, 10(12):121-123.

[Frair et al. 1998] Frair, L., Matson, J., and Matson, J. (1998). An undergraduate curriculum evaluation with the analytic hierarchy process. In Frontiers in Education Conference, 1998. FIE '98. 28th Annual, volume 3, pages 992-997 vol.3.

[Helfert and Duncan 2007] Helfert, M. and Duncan, H. (2007). Evaluating information systems and business informatics curriculum. In Proceedings of the 2007 International Conference on Computer Systems and Technologies, CompSysTech '07, pages 73:173:5, New York, NY, USA. ACM.

[Judson et al. 2015] Judson, E., Ernzen, J., Chen, Y.-C., Krause, S., Middleton, J., and Culbertson, R. (2015). What is the effect of establishing programs that address sense of belonging on undergraduate engineering retention? In Frontiers in Education Conference (FIE), 2015. 32614 2015. IEEE, pages 1-8.

[Marszalek et al. 2005] Marszalek, M., Snauffer, A., Good, S., Hein, G., and Monte, A. (2005). Mentors improve the college experience of engineering undergraduates. In Frontiers in Education, 2005. FIE '05. Proceedings 35th Annual Conference, pages F2G-F2G. 
[Monge et al. 2015] Monge, A. E., Fadjo, C. L., Quinn, B. A., and Barker, L. J. (2015). Engagecsedu: Engaging and retaining cs1 and cs2 students. ACM Inroads, 6(1):6-11.

[Oliveira Nunes et al. 2015] Oliveira Nunes, A., Veloso da Silva, T., Moura Mota, J., Lima Ferrer de Almeida, A., and Bandeira Andriola, W. (2015). Developing an instrument for assessment of academic management in engineering courses. Latin America Transactions, IEEE (Revista IEEE America Latina), 13(1):264-271.

[Outlay and Krishnan 2010] Outlay, C. N. and Krishnan, P. (2010). Skill gaps and careers in is compliance: Implications for is degree programs in the u.s. In Proceedings of the 2010 Special Interest Group on Management Information System's 48th Annual Conference on Computer Personnel Research on Computer Personnel Research, SIGMIS-CPR '10, pages 130-135, New York, NY, USA. ACM.

[Peckham et al. 2007] Peckham, J., Stephenson, P., Hervé, J.-Y., Hutt, R., and Encarnação, M. (2007). Increasing student retention in computer science through research programs for undergraduates. In Proceedings of the 38th SIGCSE Technical Symposium on Computer Science Education, SIGCSE '07, pages 124-128, New York, NY, USA. ACM.

[Satyanarayana et al. 2014] Satyanarayana, A., Li, H., and Braneky, J. (2014). Improving retention by mentoring and tutoring freshmen students. In American Society for Engineering Education (ASEE Zone 1), 2014 Zone 1 Conference of the, pages 1-5.

[Sloan 1977] Sloan, M. (1977). Evaluation of the model curriculum in computer science and engineering. Computer, 10(12):114-120.

[Thompson et al. 2014] Thompson, L. D., Eney, C., Davis, R., and Grady, T. (2014). Recruit and retain women in undergraduate computing: Success stories using research-based practices. In Proceedings of the 45th ACM Technical Symposium on Computer Science Education, SIGCSE '14, pages 541-542, New York, NY, USA. ACM. 